\title{
Effect of Driving patterns on Components sizing of a Series PHEV
}

\author{
Mitra Pourabdollah* Anders Grauers ${ }^{* *}$ Bo Egardt ${ }^{* * *}$ \\ * Chalmers University of Technology, Gothenburg, Sweden, (e-mail: \\ mitrap@chalmers.se). \\ ** Chalmers University of Technology, Gothenburg, Sweden (e-mail: \\ anders.grauers@chalmers.se) \\ *** Chalmers University of Technology, Gothenburg, Sweden, \\ (e-mail:bo.egardt@chalmers.se)
}

\begin{abstract}
In the past decade, it has been demonstrated that Plug-in Hybrid Electric Vehicles (PHEVs) can significantly reduce petroleum consumptions. However, the extend to which these vehicles can reduce the petroleum consumption highly depends on components size and driving patterns. In other words, PHEVs show the best benefits if the components are dimensioned to match the driver's driving behavior. In this paper, the effect of different driving patterns on the optimal sizing of three major components of series PHEVs, i.e., battery, electric motor, and engine generator unit is studied. Different driving cycles are generated stochastically from real driving data using Markov chains, to represent life-time driving patterns of different drivers.

To find the optimal size of the components, the problem is formulated as a convex optimization problem. The optimization variables (the variables of component size and energy management) are obtained by minimizing a cost function which is the sum of the operational and component costs.
\end{abstract}

Keywords: Hybrid vehicles, Optimal sizing, Driving cycles, Convex optimization, Markov models.

\section{INTRODUCTION}

Electrification of vehicles is becoming more and more interesting among researchers and industries because of the environmental benefits achieved by the reduction in emissions and fuel consumption. Plug-in hybrid electric vehicles (PHEVs) are the next generation of HEVs that have all the merits of HEVs gained by downsizing the engine, recovering braking energy, having extra power control freedom by the two power sources, and stopping the engine when idle. In addition, PHEVs have the additional ability to store energy from the electricity grid using large capacity batteries. PHEVs can run on the stored energy on short trips, reducing vehicle's dependency on petroleum. The extent to which this can be achieved depends highly on the size of the engine, EM, and battery as well as the driving behavior of the vehicle owner.

In car industries, components' sizing is mainly driven by performance requirements, e.g., 0-100 kph acceleration time, maximum speed, and all electric range (AER), however, in order to get the best fuel efficiency and performance, PHEVs should be designed to match the driver's driving behavior i.e., both the speed profile and the distance distribution of the trips. While the effect of single trips has been studied in detail in many papers e.g., in Moawad et al. [2009], Patil et al. [2009], Rahman et al. [1999], Gonder et al. [2007], and Kwon et al. [1999], the effect of the distance distribution of the trips has only been mentioned cursorily in Gonder et al. [2007], Frank [2007], Markel et al. [2006], Gao et al. [2010] and Smith et al. [2012]. Moreover, in most of these studies, the distribution of driving distance has been estimated per day and over the population of many drivers instead of being estimated over life-time and for single drivers. Because of huge differences in movement patterns of different drivers, there will be a huge difference in the opportunity in utilizing a PHEV Kullingsjö et al. [2012]. This means that there is a need to study how vehicles should be designed to get the best benefits for drivers with different driving behaviors.

Another aspect of PHEVs that makes the problem of sizing even more complex is energy management, i.e., the control strategy that determines how the battery should be used (or, basically, how the energy should be split between battery and engine at every time instant). Since the energy management affects both fuel economy and performance, it should ideally be part of the optimal design process in the same way as component sizing.

To find the proper design of a PHEV for different driving patterns, we cast the problem as a convex optimization problem based on quasi-static models similarly as in Pourabdollah et al. [2013]. Convexity has two important implications: (1) there is a unique optimum, and (2) fast, reliable solvers are available. This in turn means that not only component sizes but also the complete control trajectory of the energy management system can be included as optimization variables. Using convex optimization methods, the optimal sizes of the battery, EM, and EGU for the series PHEV is obtained by minimizing a cost function over a pre-specified driving cycles. The cost function comprises two parts; the first part reflects the cost of the key components of the vehicle, namely battery, EM, and EGU and the second part is the operational cost of fuel and electricity. The focus in this study is mainly on the effect of driving behavior on the components sizing. Therefore, pre-specified driving cycles are made of several stochastic trips (a trip is defined by events for which the driver has the opportunity to charge the battery) generated by a Markov process, in a way to reflect the driving behavior of different drivers. 
The constraints in the convex optimization problem are given by equations governing the power flow in the components and system, and by the maximum component ratings. Finally, the decision variables to be found by the optimization solver are the component sizes (battery, EM, and EGU) and the optimal energy management at every time instant.

The paper is organized as follows. In this Section 2, an overall picture of the optimization problem is given; we also introduce the powertrain and components model, the driving cycle, and the performance requirements used in the problem. Illustrative results from the study are shown in Section 4. Finally conclusions are drawn.

\section{PROBLEM FORMULATION}

The optimization problem that was briefly introduced in the Introduction will now be described in detail. As shown in Fig. 1, for a vehicle model and a given driving cycle, we first find the engine on-off decisions. Having these, in addition with the cost models and the performance requirements, a convex problem is solved to find the optimal component sizes and the energy management.

The objective function to be minimized in the convex optimization problem is a weighted sum of operational and component costs: $\cos t=$ cost $_{o p}+\operatorname{cost}_{\text {comp }}$.

The operational cost includes the consumed fossil fuel and electrical energy, whereas the latter includes the cost of battery, electric motor, and internal combustion engine as

$$
\operatorname{cost}_{o p}=\frac{\rho_{f}}{\rho_{L H V}} \sum_{k=1}^{N} P_{f}(k) h(k)+\frac{\rho_{e l}}{1000 \cdot 3600} \sum_{k=1}^{N} \boldsymbol{P}_{\boldsymbol{g}}(k) h(k) \text {. }
$$

where the fuel power, $P_{f}$, and charger power, $\boldsymbol{P}_{\boldsymbol{g}}$, are converted to an equivalent cost in EUR using energy prices $\rho_{f}$ for gasoline and $\rho_{e l}$ for electricity and $\rho_{L H V}$ is the lower heating value of gasoline. The time varying sampling interval $h(k)$ is equal to $1 \mathrm{~s}$ when driving and changes when charging.

The components cost is the sum of the costs of battery, EM, and EGU; the remaining cost of the vehicle is assumed to be independent of sizing and is therefore excluded from the problem. The components cost is calculated as the depreciation over the driving cycle, i.e., the proportion of the components cost given by the ratio between the length of the cycle, $d$, and the lifetime driving distance of the vehicle. Including a yearly interest rate of $p_{c}=5 \%$, the components cost is given by

$$
\operatorname{cost}_{\text {comp }}=\frac{d}{s y_{v}}\left(1+p_{c} \frac{y_{v}+1}{2}\right)\left(\operatorname{cost}_{b a t}+\operatorname{cost}_{E M}+\operatorname{cost}_{E G U}\right),
$$

where $y_{v}$ is the vehicle lifetime, and $s$ is the average traveled distance of the vehicle in one year. For each component, the cost model is a linear function as $\operatorname{cost}_{j}=\operatorname{cost}_{j, \text { init }}+\operatorname{cost}_{j, \text { slope }} \boldsymbol{s}_{j}$ for $j \in\{b a t, E M, E G U\}$, where $\boldsymbol{s}_{j}$ is a component scaling parameter used to scale the size of the components.

The decision variables of the optimization problem include, firstly, the component scaling parameters $\boldsymbol{s}_{\text {bat }}, \boldsymbol{s}_{\boldsymbol{E} \boldsymbol{M}}$, and $\boldsymbol{s}_{\boldsymbol{E} \boldsymbol{G U}}$, which are all dimensionless scaling parameters for battery, EM, and EGU. The second group consists of optimization variables which are related to the energy management and are determined for every time instant. These variables are EM torque $\boldsymbol{T}_{\boldsymbol{E} \boldsymbol{M}}(\boldsymbol{k})$, EGU power, $\boldsymbol{P}_{\boldsymbol{E G U}}(\boldsymbol{k})$, battery current, $\tilde{\boldsymbol{i}}(\boldsymbol{k})$, battery state of energy, $\boldsymbol{E}_{\boldsymbol{b}}(\boldsymbol{k})$, grid power, $\boldsymbol{P}_{\boldsymbol{g}}(\boldsymbol{k})$, and braking power, $\boldsymbol{P}_{\boldsymbol{b r k}}(\boldsymbol{k})$.

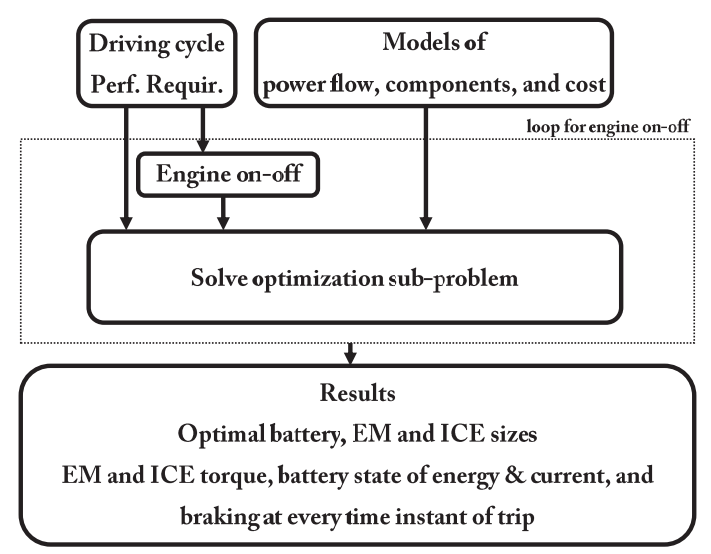

Fig. 1. Framework of the optimal PHEV design.

The optimization variables are marked in bold in the paper. The convex problem is automatically translated by a tool called CVX Grant et al. [2010] to a form required by a publicly available solver, Sedumi ${ }^{1}$. The constraints in the convex problem are in forms of powertrain and the component models, introduced in section 3 .

\subsection{Driving patterns}

The optimization finds the optimal components size and the energy management parameters over a given driving cycle. Therefore, designing a vehicle with optimal components size requires knowledge about the lifetime driving cycle of the vehicle. However, not only it is impossible to predict the precise lifetime driving cycle, but also there is a problem with the limitations of computational resources. Therefore, a short driving cycle, which still reflects the lifetime behavior of a driver is needed. As mentioned earlier, the design of Plugin Hybrid Electric Vehicle (PHEV) is influenced by both the driving distance distributions and the speed profile of the trips. In the next sections we show how we generate driving cycles considering these characteristics.

Driving distance distribution To make driving cycles that represent life-time driving cycle of different drivers, we first examine how the distribution of driving looks for different drivers from real data. We use trip distance data from 200 privately driven cars provided by the Test Site Sweden project ${ }^{2}$; the cumulative probability densities (CDF) of the trip distances for all drivers are depicted in Fig. 2.

In general, trips are separated by charging events during the parking times. Here, we assume that charging events happen once a day during night at home. Having more possibilities for charging occasions makes the trip lengths to be shorter.

To use the distributions in a systematic way, we approximate Weibull CDFs to the data. Weibull CDF is widely used in life data analysis due to its versatility and is defined as Miller et al. [2004]

$$
f(x ; \lambda, k)= \begin{cases}\frac{k}{\lambda}\left(\frac{x}{\lambda}\right)^{k-1} e^{-\left(\frac{x}{\lambda}\right)^{k}}, & x \geq 0 \\ 0, & x<0\end{cases}
$$

, with $k$ as shape parameter and $\lambda$ as scale parameters; the approximated curves to the data are shown with red curves in Fig. 2. The curves approximate the distribution of lifetime

\footnotetext{
1 Available at http://sedumi.ie.lehigh.edu/

2 Available: http://www.testsitesweden.com
} 


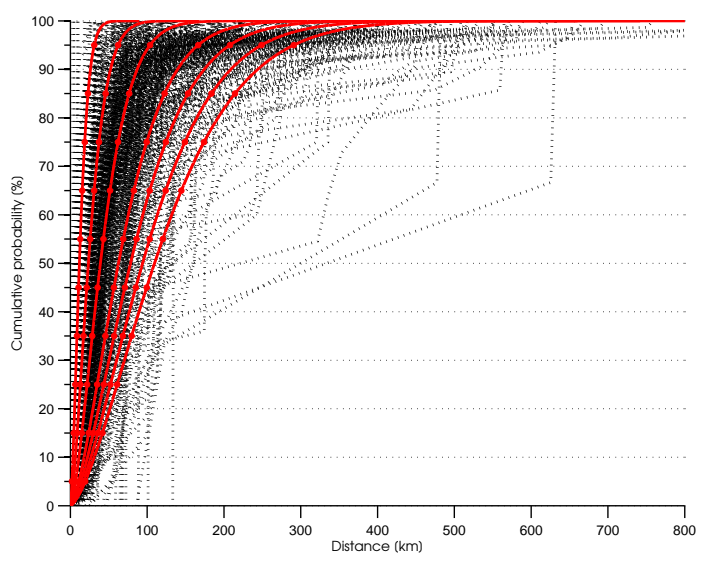

Fig. 2. The cumulative probability densities of the trip distances for 200 privately driven cars considering daily charging. Weibull cumulative distribution function for $k=1.5$ and different values of $\lambda=[15,30,50,80,100,120,140]$ are shown in red curves, and the driving lengths in the synthesized driving cycle are shown with circles.

driving distances for 7 different fictitious drivers. For example, a driver with the CDF curve similar to the Weibull curve with $\lambda=15$, drives $80 \%$ of times trips that are shorter than $23 \mathrm{~km}$, whereas for the Weibull curve with $\lambda=140$, this is less than $10 \%$. We then generate a driving cycle including 10 trips with distances taken from these $7 \mathrm{CDF}$ curves. The distances are chosen uniformly on the y-axisas as shown in Fig. 2. In this way, the trips represent similar distance distribution functions as the CDFs. However, it is worth mentioning that considering the same total driving distance for all these different drivers means that either the driver with higher values of $\lambda$ has not been driving some of the days, or the driver with lower values of $\lambda$ had more opportunities of charging, besides at nights.

Driving style A driving cycle includes the vehicle's velocity, $v(k)$, possibly road's inclination, $\beta(k)$, both at discrete time instants, and charging times between the trips. To include different driving styles and reduce the dependency of the sizing on a specific given driving cycle, stochastic trips are made from a discrete time, continuous state, time invariant Markov process. The states of the Markov process are the speed and acceleration. The initial process states are zero; the chain then moves from one state to another according to specified probabilities. The probabilities are saved in a matrix, called transition matrix, which is defined as

$$
P_{k}\left(V_{k+1}=v_{k+1}, A_{k+1}=a_{k+1} \mid v_{k}, a_{k}\right)
$$

for all possible combinations of $v_{k+1}$ and $a_{k}$. To construct the transition matrix, we use the real data from the Test Site Sweden project. Since the style of driving can be different for different trip distances, we divide the data into different groups so that we get different transition matrices for each; the procedure is similar as given in Lee et al. [2011]. Here, the driving cycles are divided into three groups, city (distance $\leq 5)$, mixed $(5<$ distance $\leq 30)$ and highway $(30<$ distance). The properties of these cycles are given in Table 1. Later, for a desired trip distance, the corresponding transition matrix is used to generate a stochastic driving cycles.
Table 1. Parameters of the cycle

\begin{tabular}{llll}
\hline parameter & city & mixed & highway \\
number of trips & 943 & 481 & 208 \\
distance & $5765 \mathrm{~km}$ & $8293 \mathrm{~km}$ & $7269 \mathrm{~km}$ \\
time $(\mathrm{N})$ & $194 \mathrm{~h}$ & $164 \mathrm{~h}$ & $97 \mathrm{~h}$ \\
max. speed & $120 \mathrm{kph}$ & $123 \mathrm{kph}$ & $123 \mathrm{kph}$ \\
average speed & $30 \mathrm{kph}$ & $50 \mathrm{kph}$ & $74 \mathrm{kph}$ \\
max. acceleration & $4.8 \mathrm{~m} / \mathrm{s}^{2}$ & $4.8 \mathrm{~m} / \mathrm{s}^{2}$ & $4.7 \mathrm{~m} / \mathrm{s}^{2}$ \\
avrg. acceleration & $0.4 \mathrm{~m} / \mathrm{s}^{2}$ & $0.3 \mathrm{~m} / \mathrm{s}^{2}$ & $0.2 \mathrm{~m} / \mathrm{s}^{2}$ \\
max. deceleration & $5.6 \mathrm{~m} / \mathrm{s}^{2}$ & $4.9 \mathrm{~m} / \mathrm{s}^{2}$ & $4.9 \mathrm{~m} / \mathrm{s}^{2}$ \\
\hline
\end{tabular}

\subsection{Performance requirements}

A long driving cycle can reflect real-life driving, but might not include extreme situations that require high performance. However, high performance is still considered as an important vehicle attribute by many drivers. In series HEVs, it is only the EM that directly drives the vehicle, therefore we can incorporate such performance requirements as explicit constraints on the EM size. Here, we have considered the maximum speed of $160 \mathrm{kph}$, constant speed of $90 \mathrm{kph}$ at $10 \%$ slope, and 0 $100 \mathrm{kph}$ acceleration time of 9 seconds as the performance requirements. Using the baseline vehicle mass, these performance requirements demand a minimum size of the EM equal to $73.5 \mathrm{kWh}$. Moreover, the sum of the power from EGU and battery need to provide the maximum power demand of the EM.

\section{MODELING}

Modeling of the powertrain and its components is a crucial step of the optimization method, since convexity has to be guaranteed. This section presents the powertrain model and the component models.

\subsection{Powertrain model}

The studied PHEV, depicted in Fig. 3, is a powertrain with series topology where only the EM is mechanically linked to the drive train and can propel the wheels. The engine generator unit (EGU) can directly feed the EM or charge the battery. Having the velocity $v(k)$, the acceleration $a(k)$, and the road slope $\beta(k)$ at discrete time instants $k$ from the driving cycle, the required traction force $F_{t}(k)$ can be calculated as

$F_{t}(k)=\frac{c_{d} A_{f} \rho v(k)^{2}}{2}+m_{t o t} g c_{r} \cos (\beta(k))+m_{t o t} g \sin (\beta(k))+m_{t o t} a(k)$,

where $F_{t}$ is the longitudinal force from the drive line and $m_{t o t}, A_{f}, c_{d}, \rho, g, c_{r}, \beta$, and $a$ are total vehicle mass, frontal area, air drag coefficient, air density, gravitational acceleration, rolling resistance coefficient, road angle, and acceleration, respectively. The total vehicle mass, $m_{t o t}$, is the sum of the masses of the glider, battery, EM, and EGU, which are linear functions of sizes, or $m_{j}=m_{j, \text { slope }} \boldsymbol{s}_{j}$ for $j \in\{$ bat, $E M, E G U\}$.

The powertrain model is described by power balance equations, given as

$$
P_{d e m}(k)+P_{b r k}(k)=\boldsymbol{T}_{\boldsymbol{E M}}(\boldsymbol{k}) \omega_{E M}(k),
$$

$$
\omega_{E M}(k) \boldsymbol{T}_{\boldsymbol{E M}}(\boldsymbol{k})+P_{E M, l o s s}(k)=\boldsymbol{P}_{\boldsymbol{E G U}}+P_{b a t}(k)-P_{a u x},
$$
with $P_{d e m}(k)$ calculated from (5); $\boldsymbol{P}_{\boldsymbol{b r k}}$ is the power dissipated at the friction brakes; $\boldsymbol{T}_{\boldsymbol{E M}}, \omega_{E M}$ and $P_{E M \text {,loss }}$ are the torque, speed and power losses of the EM; $P_{b a t}, \boldsymbol{P}_{\boldsymbol{E G U}}$, and $P_{a u x}$ are the battery power, the mechanical power of the EGU and the 


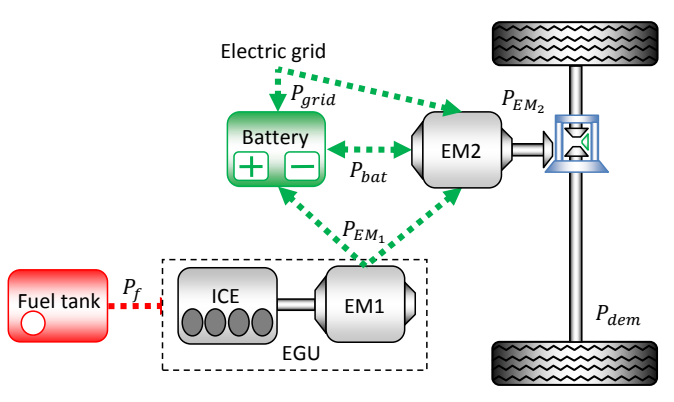

Fig. 3. Series PHEV configuration (solid lines: mechanical link, and dashed lines: electrical links).

Table 2. Vehicle parameters

\begin{tabular}{ll}
\hline \hline parameter & value \\
\hline baseline mass $(\mathrm{m})$ & $1600 \mathrm{~kg}$ \\
glider mass $\left(m_{g}\right)$ & $1280 \mathrm{~kg}$ \\
frontal area $($ Af) & $2.37 \mathrm{~m}^{2}$ \\
rolling resistance $\left(c_{r}\right)$ & 0.009 \\
aerodynamic drag coefficient $\left(c_{d}\right)$ & 0.33 \\
air density $(\rho)$ & $1.293 \mathrm{~kg} / \mathrm{m}^{3}$ \\
wheel radius $\left(r_{w}\right)$ & $0.3 \mathrm{~m}$ \\
ratio of the final gear $\left(r_{f g}\right)$ & 4.2 \\
EM reduction gear $\left(r_{E M}\right)$ & 2 \\
\hline
\end{tabular}

electrical power used by auxiliary devices. For simplicity, the losses in the power electronics are included in the EM losses and the rotational inertia (including the inertia of the wheels, the differential, the EM and the EGU) are neglected in the models. The vehicle's parameters are given in Table 2. At each time instant on the driving cycle, the angular speed of the EM is calculated in advance as

$$
\omega_{E M}(k)=r_{E M} v(k) \frac{r_{f g}}{r_{w}},
$$

where $r_{w}, r_{f g}$, and $r_{E M}$ are the wheel radius, ratio of the final gear (differential), and EM reduction gear respectively.

\subsection{Battery}

The battery consists of $\boldsymbol{s}_{\text {bat }}$ identical cells, each modeled as an open circuit voltage, $V_{o c}$, in series with a constant internal resistance, $R$. The terminal power, $P_{b a t}$, and the stored energy of the battery, $E_{b}$, are calculated as

$$
\begin{gathered}
P_{b a t}(k)=\boldsymbol{s}_{\boldsymbol{b a t}}\left(V_{o c} i(k)-R i^{2}(k)\right)=V_{o c} \tilde{\boldsymbol{i}}(\boldsymbol{k})-R \frac{\tilde{\boldsymbol{i}}^{\mathbf{2}(\boldsymbol{k})}}{\boldsymbol{s}_{\boldsymbol{b a t}}} \\
\boldsymbol{E}_{\boldsymbol{b}}(\boldsymbol{k}+\mathbf{1})=\boldsymbol{E}_{\boldsymbol{b}}(\boldsymbol{k})-h(k) V_{o c} \tilde{\boldsymbol{i}}(\boldsymbol{k}) .
\end{gathered}
$$

The cell current $i(k) \in\left[i_{\text {min }}, i_{\text {max }}\right]$ is chosen to be positive when discharging and the current $\tilde{\boldsymbol{i}}(\boldsymbol{k})=\boldsymbol{s}_{\text {bat }} i(k)$ is a variable change that is introduced to keep the convexity [Murgovski et al., 2012]. The voltage over each cell, $V_{o c}$, is approximated to be constant; this assumption can be justified when operating in limited SoC ranges. During the available parking periods, $\Delta t_{c}(k)$, it is assumed without loss of generality that the vehicle is charged with constant current and power. Then, it is assumed that the whole charging energy enters the battery in one extra long sample, $\Delta t_{c}(k)$, at the parking events. In this way, at charging occasions in (10), $h(k)$ is equal to $\Delta t_{c}(k)$.

\subsection{Electric motor}

The EM model with its power electronics is described by a power loss map, $P_{E M \text {,loss,base, }}$, where the losses are measured at steady-state for different torque-speed combinations. The power losses for each EM speed can be approximated by a second-order polynomial in the torque

$$
\begin{aligned}
& P_{E M, \text { loss,base }}\left(\omega_{E M}, T_{E M, \text { base }}\right)= \\
& c_{1}\left(\omega_{E M}\right) T_{E M, \text { base }}^{2}+c_{2}\left(\omega_{E M}\right) T_{E M, \text { base }}+c_{3}\left(\omega_{E M}\right)
\end{aligned}
$$

where the coefficients $c_{1} \geq 0, c_{2}$ and $c_{3}$ functions of $\omega_{E M}$ and are calculated using least squares method [Murgovski et al., 2012]. To vary the size of the EM, the maximum and minimum torque, $T_{E M, \text { max,base }}\left(\omega_{E M}(k)\right)$ and $T_{E M, \text { min,base }}\left(\omega_{E M}(k)\right)$, are scaled by the scaling factor $\boldsymbol{s}_{\boldsymbol{E M}}$. The losses are also assumed to change linearly with $\boldsymbol{s}_{\boldsymbol{E} \boldsymbol{M}}$. Hence, given a baseline EM described by torque $T_{\text {em,base }}$ and losses $P_{E M \text {,loss, base }}$, the losses of the scaled EM are calculated at each time instant as

$$
P_{E M, l o s s}(k)=c_{1}(k) \frac{\boldsymbol{T}_{\boldsymbol{E M}}^{\mathbf{2}}(\boldsymbol{k})}{\boldsymbol{s}_{\boldsymbol{E} \boldsymbol{M}}}+c_{2}(k) \boldsymbol{T}_{\boldsymbol{E M}}(\boldsymbol{k})+c_{3}(k) \boldsymbol{s}_{\boldsymbol{E M}} \text {. }
$$

This nonlinear model is convex in $\boldsymbol{T}_{\boldsymbol{E M}}$ and $\boldsymbol{s}_{\boldsymbol{E} \boldsymbol{M}}$ for $\boldsymbol{s}_{\boldsymbol{E M}}>0$.

\subsection{Engine generator unit}

The fuel power, $P_{f}$, of an EGU is a function of the generator power and is approximated with a second-order polynomial in $P_{E G U}$ as

$$
P_{f, \text { base }}\left(P_{E G U}\right)=b_{1} P_{E G U, \text { base }}^{2}+b_{2} P_{E G U, \text { base }}+b_{3}
$$

where $b_{j} \geq 0 ; j \in 0,1,2$, and are calculated in a similar way as $c_{1}, c_{2}$, and $c_{3}$ for the EM [Murgovski et al., 2012]. Considering that losses vary linearly with respect to scaling factor $\boldsymbol{s}_{\boldsymbol{E} \boldsymbol{G} U}$, the fuel power is calculated as

$$
P_{f}(k)=b_{1} \frac{\boldsymbol{P}_{\boldsymbol{E G U}}^{\boldsymbol{2}}(\boldsymbol{k})}{\boldsymbol{s}_{\boldsymbol{E} \boldsymbol{G U}}}+b_{2} \boldsymbol{P}_{\boldsymbol{E} \boldsymbol{G U}}(\boldsymbol{k})+e_{o n}(k) b_{3} \boldsymbol{s}_{\boldsymbol{E} \boldsymbol{G U}} .
$$

where the variable $e_{o n}$ is introduced to remove the idling losses $b_{3} s_{E G U}$, when the EGU is off. The decision variable $e_{\text {on }}$ is made prior to the optimization to preserve the problem convexity and it is decided based on the baseline power demand required by the vehicle when following the driving cycle.

\section{RESULTS}

The optimization problem gives the optimal components size over a given driving cycle therefore, we study the effect of different driving patterns on the components sizing. Since driving cycles are combination of trips with different lengths, to fully understand the effect, we first find the optimal components size of PHEVs assuming that the driver drives only a single trips with a specific length. We use the results to explain the effect of the driving cycles that represent different life time driving behaviors on component sizing.

\subsection{Single trips with fixed length}

Stochastic trips have been generated with trip lengths from 1 to $180 \mathrm{~km}$. To see the effect of different speed profiles, for each trip length we use 10 stochastic trips. The optimal sizes of battery, EM, and EGU at each trip length are shown in Fig 4. The battery size is shown by its power, however, there is a linear function between the power and capacity, i.e., a battery with capacity of $1 \mathrm{kWh}$ has a power of $5.53 \mathrm{~kW}$. 

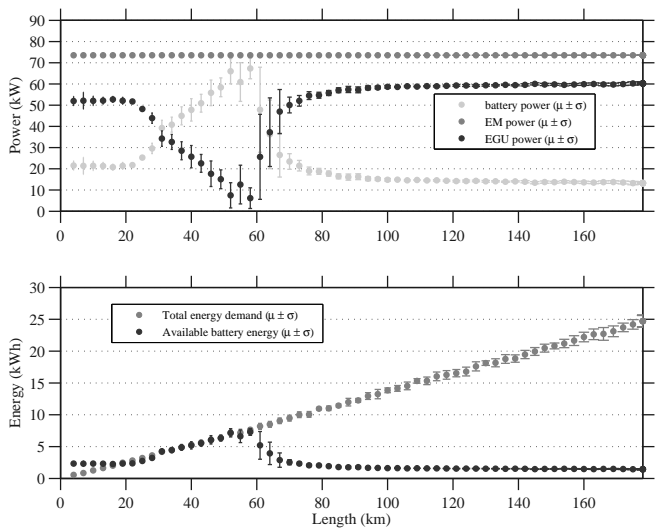

Fig. 4. Optimal battery, EM, and EGU size for single trips with different lengths.

As can be seen, the EM has constant size for all the cycles, which is decided by the performance requirements (the second order effect due to the change of weight is neglected due to the use of the baseline weight). This is because the power demand of the performance requirement has been higher than the power demand from the trips. For short trips up to $22 \mathrm{~km}$, the battery and EGU have constant sizes of around $20 \mathrm{kw}$ and $52 \mathrm{kw}$ respectively. Considering the energy demand of the trips, we can see that the battery is oversized from the energy point of view. This is due to power limitations and resistance losses, which increase/decreases respectively by increasing the number of battery cells. In other words, if the battery had no power limitation, the EGU size would become zero, since it is only needed at high power demands. On the other hand, if the battery resistance was smaller, the battery would have been sized by the energy demand, which increases approximately linearly by the trip distance.

For trips with lengths between 25 and $60 \mathrm{~km}$, the battery power/size increases because of the increasing energy demand; increase in battery capacity results in higher battery power, which in turn decreases the need on the power from EGU, therefore the EGU becomes smaller. For trips with approximate length of $60 \mathrm{~km}$, it is almost optimal to have an electric vehicle. However, for longer trips, the optimal battery size suddenly drops which makes the optimal vehicle become more similar to a HEV than EV. This is because it is not cost efficient anymore to increase the size of a heavy and costly battery, to drive all electrically; instead, the battery is sized small to be used for having extra freedom and recovering the braking energy. In this way, since the energy charged from the grid is not much, the EGU can operate most of the time in higher power, which means higher efficiency.

One important thing to notice here is that different driving styles with same trip distance do not effect the sizing significantly. However, this happens only when we add the constraints from the performance requirements. For the cases where these constraints are relaxed, the sizes vary much for different trips. The reason is mainly because these trips are more different in power than energy, and when the power is limited by performance requirement (which is similar for all trips), the sizing becomes less effected by the trips, and more by the trip lengths or energy.

It is important to mention that the optimization can not give the extreme cases, i.e. a conventional vehicle with no battery and

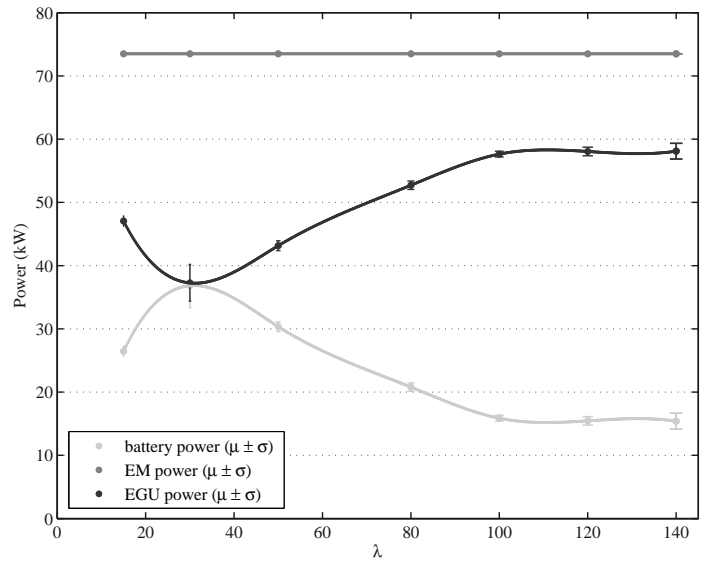

Fig. 5. Average optimal size of components for different driving cycles, corresponding to different values of $\lambda$.

an electric vehicle without an EGU. This is because the convex cost function of these components are linear with an offset (the packaging cost). With this cost model, even if the sizes are zero, the cost will include the packaging cost. Therefore, to exclude the total cost of battery or EGU, the optimal has to be run separately including zero cost for these components.

\subsection{Trips representing life-time driving}

Having examined how the trip length effects the sizing, we can now study how combination of these trips effect the optimal components size. We generate driving cycles which are combination of 10 trips with different length with probabilities according to the CDF curves in Fig 2.

The result of the optimal components size for driving cycles corresponding to different values of $\lambda$ are shown in Fig 5. To get more general results, and see the effect of different driving styles, we have made 10 different driving cycles for each CDF. However, similarly to single trips cases, the sizes are quite robust to the differences in speed profiles. Therefore, in Fig 5, only the mean values of the sizes are shown.

For a driver who drives very short distances most of the times (the driving cycle corresponding to the CDF with $\lambda=15$ ), the optimal battery size is quite small, due to small energy demand. As the driver drives longer distances (the driving cycle corresponding to the CDF with $\lambda=30$ ), the optimal battery size increases. As mentioned in the previous section, this is due to the increasing energy demand on most cycles. However, for driving cycles corresponding to larger values of $\lambda$, the battery size starts decreasing. This happens because cycles with lengths longer than $60 \mathrm{~km}$, for which the optimal battery size is rather small, become more influential. Eventually, for driving cycles corresponding to $\lambda>100$, the battery is sized by the dominant trips with distances longer than $60 \mathrm{~km}$, occurring more than $50 \%$ of the time. This is in accordance with the results for single trips longer than $60 \mathrm{~km}$, where the optimal battery size is approximately $3 \mathrm{kWh}$.

\section{CONCLUSION}

In this paper, we use convex optimization techniques to solve the problem of sizing the three major components of a plug-in hybrid electric vehicle, i.e. the battery, the electric motor, and the engine generator unit. To define the problem as a convex 
optimization problem, the model of the vehicle and components are approximated as convex functions. We mainly focus on the effect of driving behaviors on component sizing. The driving cycles used in the optimization are generated stochastically by Markov chains, to represent behavior of drivers with different driving distance distributions.

The results show that the size of the EM for a series PHEV is decided by performance requirements. To provide the enough power to the EM, the sum of EGU and battery power has to be equal to or higher than the power of the EM. The size of the engine generator unit and battery, however, are primarily affected by the distribution of trip lengths. For instance, for a driver that often drives short distances, a vehicle with a small battery is optimal. If the driver drives longer distances, the optimal battery size increases, while the EGU size decreases. these characteristics are similar to the characteristics of an electric vehicles. However, as the trips get even longer, the optimal battery size decrease suddenly, as for an HEV.

In this particular study, we have specifically focused on the distribution of trip lengths. We have made several driving cycles stochastically from real data in order to gain general results in the sense that they are not dependent on a specific trip. However, these synthesized trips should be analyzed in greater detail to verify that they include most of the real life driving conditions. We have also used only an energy optimized battery, which is more appropriate for PHEV or EV vehicles, whereas for HEVs, an energy optimized battery is more suitable. Future work can also include using different battery types with different prices.

\section{ACKNOWLEDGMENT}

The simulations were performed on resources provided by the Swedish National Infrastructure for Computing (SNIC) at C3SE.

\section{REFERENCES}

A. Moawad, G. Singh, S. Hagspiel, M. Fellah, A. Rousseau, "Impact of Real World Drive Cycles on PHEV Fuel Efficiency and Cost for Different Powertrain and Battery Characteristics," EVS 24 Stavanger, Norway, May 13-16, 2009.

R. Patil, B. Adornato and Z. Filipi, "Impact of Naturalistic Driving Patterns on PHEV Performance and System Design," SAE Technical Paper 2009-01-2715, 2009.

Z. Rahman, K. Butler, and M. Ehsani, "A Study of Design Issues on Electrically Peaking Hybrid Electric Vehicle for Diverse Urban Driving Patterns," Advances in electric Vehicle Technology (SAE-SP-1417). Warrendale, SAE, 1999, p. 27-35.

J. Gonder, T. Markel, A. Simpson, and M. Thornton, "Using GPS Travel Data to Assess the Real World Driving Energy Use of Plug-In Hybrid Electric Vehicles (PHEVs)," Transportation Research Board (TRB) 86th Annual Meeting, Washington D.C., January 21-25, 2007.

J. Kwon, J. Kim, E. Fallas, S. Pagerit, A. Rousseau, "Impact of Drive Cycles on PHEV Component Requirements,' SAE Technical Paper 2008-01-1337, 2008.

A. A. Frank, "Plug-in Hybrid Vehicles for a Sustainable Future,' U.S. Department of Transportation, Am. Sci. 95(2), pp. 158165.

T. Markel, A. Brooker, J. Gonder, M. OKeefe, A. Simpson, and M. Thornton, "Plug-in Hybrid Vehicle Analysis," National
Renewable Energy Laboratory, Report No. NREL/MP-54040609.

Y. Gao and M. Ehsani,, "Design and Control Methodology of Plug-in Hybrid Electric Vehicles," IEEE Trans. Ind. Electron., vol. 57, no. 2, Feb. 2010.

K. Smith, M. Earleywine, E. Wood, J. Neubauer, and A. Pesaran, "Comparison of Plug-In Hybrid Electric Vehicle Battery Life Across Geographies and Drive Cycles," SAE Technical Paper 2012-01-0666, 2012.

L. H. Kullingsjö, S. Karlsson, "The Swedish car movement data project," Proceedings from EEVC, Brussels, Belgium, November 19-22, 2012.

M. Pourabdollah, N. Murgovski, A. Grauers, and B. Egardt, "Optimal sizing of a parallel PHEV powertrain," IEEE Trans. on Veh. Tech., vol. PP, no. 99, Feb. 2013.

S. Miller, D. Childers, Probability and Random Processes: With Applications to Signal Processing and Communications, Elsevier, 2004.

T. K. Lee , Z. S. Filipi, Synthesis of real-world driving cycles using stochastic process and statistical methodology, Int. J. Vehicle Design, vol. 57, no. 1, 2011.

N. Murgovski, L. Johannesson, J. Sjöberg, B. Egardt, “Component sizing of a plug-in hybrid electric powertrain via convex optimization," J. Mechatronics, vol. 22, no. 1, pp. 106-120, Feb. 2012.

M. Grant and S. Boyd (2010, May), CVX: Matlab software for disciplined convex programming, version 1.21 [Online]. Available: http://cvxr.com/cvx 